\title{
Measures for the Quality of Individual Articles, Authors and Journals
}

\author{
R. Rajesh, K. Rajeev, N.K. Ragesh, V. Gopakumar \\ TDC, Network Systems and Technologies (P) Ltd. \\ Technopark, Thiruvananthapuram \\ Kerala - 695581, India
}

\begin{abstract}
Journal impact factors are not representatives of the quality measures of individual journal articles, authors and quality factor of journals. Hence, due to the high necessity of quality measures, this paper presents new quality measures for journals, authors and individual journal articles.
\end{abstract}

\section{General Terms}

Quality of Paper (Q), Author Quality Factor (AQF), Quality of Journal (J), Science Citation Index (SCI), Impact Factor, Journal Citation Reports (JCR).

\section{Keywords}

Quality of Paper (Q), Author Quality Factor (AQF), Quality of Journal (J), Science Citation Index (SCI), Impact Factor, Journal Citation Reports (JCR).

\section{INTRODUCTION}

Evaluating scientific quality of papers/individuals is a difficult problem and so far no standard solution has been found out. Hence individuals for evaluating their research depend on secondary criteria like crude publication counts in citation data. The citation data are obtained from a database produced by the Institute for Scientific Information (ISI) in Philadelphia, which continuously records scientific citations as represented by the reference lists of articles from a large number of scientific journals and the results are published as the Science Citation Index (SCI). The citation rate of a scientific journal is known as the journal impact factor and can be calculated as the mean citation rate of all the articles contained in the journal [8]. Lot of literature has appeared which favors and criticizes impact factor [6], [7], [9], [13], [14], [15], [16], [19], [20], [21], [22], [23]. There are more indices, many more of them, including variants of those above that take into account the age of papers or the number of authors [1], [18], [17].

Thomson Reuters encourages everyone within the research community to do their parts in preventing misunderstanding and misuse of the Journal Impact Factor and they clearly suggest (1) not to use the Journal Impact Factor to assess the performance of an individual researcher, and (2) as with all of the metrics provided through Journal Citation Reports (JCR), the Journal Impact Factor should only be used to compare journals.

It should also be noted that Journal impact factors are not representatives of the quality measure of individual journal articles and the quality factor of journals. Hence there is a high necessity of quality index for individual journal articles, authors and journals. Hence this paper presents new quality measures for journals, authors and individual journal articles. These quality measures should be provided in the individual articles so as to give good transparency in the evaluating process and also to help in judging the research articles, authors and journals.

\section{COMMONLY USED INDICES - A REVIEW}

Impact factor of a journal, in a given year, is the average number of citations to those papers that were published during the two preceding years [10]. For example, the 2010 impact factor of a journal would be calculated as $\mathrm{A} / \mathrm{B}$, where $\mathrm{A}$ is the number of times articles published in 2009 \& 2008 were cited by indexed journals during 2010, and B is the total number of "citable items" published in $2008 \& 2009$.

Journal Citation Report (JCR) also includes the Eigenfactor Metrics [3], which use citing journal data from the entire JCR file to reflect the prestige and citation influence of journals by considering scholarly literature as a network of journal-to-journal relationships.

In addition to the collaboration with the University of Washington to include Eigenfactor Metrics, the Journal Citation Reports (JCR) includes the following new features:

1) Five-Year Impact Factor.

2) An analysis of journal self citations and their contribution to the Journal Impact Factor calculation.

3) A graphic interpretation (Box Plot) of how a journal ranks in different categories.

4) Rank-in-Category Tables for Journals Covering Multiple Disciplines.

Another useful index to characterize the scientific output of a researcher is the h-index proposed by Jorge E. Hirsch, Professor of Physics at the University of California [2], [5], [11], [12], [4]. A scientist has an index $h$, if $h$ of his or her $N p$ papers have at least $h$ citations each and the other $(N p-h)$ papers have less than or equal to $h$ citations each.

The $h$ index does not take into account the fact that some papers in the top $n$ may have extraordinarily high citation counts. The $g$ index, proposed by Leo Egghe, is meant to compensate for this. A scientist's $g$ index is the largest $n$ for which the $n$ most cited papers have a total of at least $n^{2}$ citations. 


\section{NEW QUALITY FACTORS}

Published scientific results are usually scrutinized by true experts in the field based on the quality factors but not limited to the following.

1) Relevance $\left(q_{1}\right)$ - Is the topic of the paper relevant to the scope of journal and its wide readers? Does it show the potential to stimulate high end research?

2) Originality $\left(q_{2}\right)$ - How novel and innovative is the paper? A paper presenting methods or application domains not frequently discussed will receive a high mark. This also takes into consideration whether the topic has been published in similar form before. If the paper contains mostly known material, i.e. established methods and well understood application domains, it is not considered very original. Empirical case studies of a particular application domain are often highly original, but may have only limited significance to the field.

3) Significance $\left(q_{3}\right)$ - Does the paper make a valuable contribution to the theory or the practice? It represents an indicator of the importance of the findings, regardless of their degree of originality.

4) Content $\left(q_{4}\right)$ - What is the information content of the paper? Does the paper allow non-experts in the field to comprehend its research objective?

5) Soundness $\left(q_{5}\right)$ - Is the paper technically correct (considering its submission category)? What is the technical quality? Were all technical and technological aspects of the experiments well documented? (reliability) Were results compared to established benchmark practices, methods etc.? Were the results evaluated taking care of established standard procedures (validity)? Moreover soundness will take care of quality of literature review, statement of research goals, appropriate use of the most relevant references to indicates orientation within the field, documented methods, logical presentation, analysis of results, findings, inferences and conclusions.

6) Clarity $\left(q_{6}\right)$ - Is the paper well presented and organised? A well presented paper enhances the understanding of the presented content also to non experts in the field. It often shows clear and logical presentation, appropriate style, the standard of English, freedom from errors, ease of reading, correct grammar and spelling, appropriate abstract, adequate use of graphical materials and tables to support ideas \& findings, conformance with journal specifications for referencing, length and format details.

The above six quality factors should be evaluated on five point scale with values (very low (0), low (0.5), medium (1), high (1.5), very high (2)). The individual quality factors are not all of the same importance and may be weighted to provide a final score. Let $w_{1}, w_{2}, w_{3}, w_{4}, w_{5}, w_{6}$ be the weights assigned for the quality factors. Then the rating of the paper by the $i^{\text {th }}$ reviewer can be calculated as

$$
V_{i}=\frac{\sum_{j=1}^{6} q_{j} w_{j}}{\sum_{j=1}^{6} w_{j}}
$$

where $q_{1}, q_{2}, q_{3}, q_{4}, q_{5}, q_{6}$ be the values assigned for the quality factors by $\mathrm{i}^{\text {th }}$ reviewer.

A reviewer's expertise for a topic indicates how familiar she/he is with current research, publications, best practices and applications in the field. Reviewers with a high confidence will be able to evaluate a paper more accurate than a reviewer with little expertise in the field. Reviewers expertise/confidence (E) will also be on five point scale with values (very low (0), low $(0.5)$, medium (1), high (1.5), very high (2)).

Let $\mathrm{F}$ be the impact factor of a journal, $h_{i}$ be the h-index of the $\mathrm{i}^{\text {th }}$ author, $g_{i}$ be the g-index of the $\mathrm{i}^{\text {th }}$ reviewer, $E_{i}$ be the $\mathrm{i}^{\text {th }}$ reviewer's expertise, $V_{i}$ be the rating of the paper by $\mathrm{i}^{\text {th }}$ reviewer, $C_{i}$ be the number of citations of $\mathrm{i}^{\text {th }}$ paper, $Q$ be the quality a paper, $A Q F$ be the author quality factor, and $J$ be the quality a journal. Let

$$
R_{i}=1+\frac{1}{10} \log \left(1+g_{i}\right)
$$

where $g_{i}$ is the g-index of the $\mathrm{i}^{\text {th }}$ reviewer.

Definition 1. The quality of the paper $(Q)$ can be calculated as

$$
Q=\left(\sum_{i=1}^{n} V_{i}^{R_{i} E_{i}}\right) \times\left(1+\log \left(1+\frac{1}{m} \sum_{j=1}^{m} h_{j}\right)\right)
$$

where $n$ is the number of reviewers, $m$ is the number of authors in the paper, and $h_{j}$ is the h-index of the $j^{\text {th }}$ author.

Definition 2. Evaluation of authors can be done using author quality factor $(A Q F)$ given by

$$
A Q F=\log \left(1+\sum_{i=1}^{p}\left(Q_{i} \times\left(1+C_{i}\right)\right)\right)
$$

where $p$ is the number of papers published by the author, $Q_{i}$ is the quality of $i^{\text {th }}$ paper, and $C_{i}$ is the number of citations of $i^{\text {th }}$ paper.

Definition 3. The quality of single issue of a journal (J) is given by

$$
J=\left[\log \left(1+(1+F) Q_{T}\right)\right] \times \frac{1}{k} \sum_{i=1}^{k} R_{i}
$$

where (i) $k$ is the number of reviewers of the journal including Editor-in-chief, Editors, and Associate Editors, (ii) $F$ is the impact factor of the journal, (iii) $Q_{T}=\sum_{j=1}^{m} Q_{j}$, where $m$ is the number of papers published.

Remark: - The five-year quality of the journal can be calculated by finding the average of the qualities of all the issues of the journal for the last five years.

On providing these measures (like paper rating $\left(V_{i}\right)$, quality of paper $(Q)$, quality of journal $(J), g$ index of the reviewers, $h$ index of the authors of the papers) in each articles in all journals in the world will give good transparency in judging the research articles, authors and journals. The space and cost required to provide these measures in each research article is very negligible and is affordable to any publishers. 


\section{ILLUSTRATION}

\subsection{Evaluation of Quality of Paper}

Let table I show the evaluation results of a paper submitted by three authors with h-index $h_{1}=1, h_{2}=10$, and $h_{3}=3$. Let the reviewers expertise on the subject relevant to the paper submitted be $E_{1}=1, E_{2}=1.5, E_{3}=2$. Let the g-index of first, second and third reviewer be $g_{1}=1, g_{2}=5, g_{3}=12$ respectively.

Let the weights assigned to $q_{1}, q_{2}, q_{3}, q_{4}, q_{5}, q_{6}$ be $w_{1}=1, w_{2}=2$, $w_{3}=1, w_{4}=4, w_{5}=1, w_{6}=1$ respectively. Then

$$
\begin{aligned}
& V_{1}=\frac{0.5 \times 1+1 \times 2+1.5 \times 1+1.5 \times 4+1 \times 1+0.5 \times 1}{1+2+1+4+1+1}=1.15 \\
& V_{2}=\frac{1 \times 1+2 \times 2+2 \times 1+1.5 \times 4+1 \times 1+1 \times 1}{1+2+1+4+1+1}=1.5 \\
& V_{3}=\frac{2 \times 1+2 \times 2+1.5 \times 1+0.5 \times 4+0.5 \times 1+1 \times 1}{1+2+1+4+1+1}=1.1 \\
& R_{1}=1+\frac{1}{10} \log (1+1)=1.0693 \\
& R_{2}=1+\frac{1}{10} \log (1+5)=1.1792 \\
& R_{3}=1+\frac{1}{10} \log (1+12)=1.2565
\end{aligned}
$$

Then the Quality of the paper,

$$
\begin{gathered}
Q=\left(1.15^{1.0693^{* 1}}+1.5^{1.1792 * 1.5}+1.1^{1.2565^{* 2}}\right) \\
\times\left(1+\log \left(1+\frac{1}{3}(1+10+3)\right)\right)=12.2523
\end{gathered}
$$

Table 1. Review results of Paper 1

\begin{tabular}{|c|l|c|c|c|}
\hline No. & Quality factors & $\begin{array}{c}\mathbf{1}^{\text {st }} \text { Review } \\
\mathbf{E}_{\mathbf{1}}=\mathbf{1}\end{array}$ & $\begin{array}{c}\mathbf{2}^{\text {nd }} \text { Review } \\
\mathbf{E}_{\mathbf{2}}=\mathbf{1 . 5}\end{array}$ & $\begin{array}{c}\mathbf{3}^{\text {rd }} \text { Review } \\
\mathbf{E}_{\mathbf{3}}=\mathbf{2}\end{array}$ \\
\hline 1 & Relevance $\left(\mathrm{q}_{1}\right)$ & 0.5 & 1 & 2 \\
\hline 2 & Originality $\left(\mathrm{q}_{2}\right)$ & 1 & 2 & 2 \\
\hline 3 & Significance $\left(\mathrm{q}_{3}\right)$ & 1.5 & 2 & 1.5 \\
\hline 4 & Content $\left(\mathrm{q}_{4}\right)$ & 1.5 & 1.5 & 0.5 \\
\hline 5 & Soundness $\left(\mathrm{q}_{5}\right)$ & 1 & 1 & 0.5 \\
\hline 6 & Clarity $\left(\mathrm{q}_{6}\right)$ & 0.5 & 1 & 1 \\
\hline
\end{tabular}

\subsection{Evaluation of Author Quality Factor}

Let an author have 10 papers with quality of the paper and number of citations of the paper as follows.

$$
\begin{aligned}
& Q_{1}=3.2343 \quad Q_{2}=3.9613 \quad Q_{3}=4.5135 \\
& Q_{4}=5.9316 \quad Q_{5}=6.1071 \quad Q_{6}=7.4454 \\
& Q_{7}=1.8377 \quad Q_{8}=2.2122 \quad Q_{9}=0.0746 \\
& Q_{10}=1.3863 \\
& C_{1}=5 \quad C_{2}=0 \quad C_{3}=1 \quad C_{4}=10 \quad C_{5}=0 \\
& C_{6}=3 \quad C_{7}=2 \quad C_{8}=0 \quad C_{9}=1 \quad C_{10}=0
\end{aligned}
$$

Then the Author Quality Factor,

$$
\begin{aligned}
A Q F= & \log [1+3.2343 *(1+5)+3.9613 *(1+0)+4.5135 *(1+1) \\
& +5.9316 *(1+10)+6.1071 *(1+0)+7.4454 *(1+3) \\
& +1.8377 *(1+2)+2.2122 *(1+0)+0.0746 *(1+1) \\
& +1.3863 *(1+0)]=4.9684
\end{aligned}
$$

\subsection{Evaluation of Quality of a Single Issue of a Journal}

Let an issue of a journal have 10 papers with quality of the paper as follows.

$$
\begin{array}{lll}
Q_{1}=3.2343 & Q_{2}=3.9613 & Q_{3}=4.5135 \\
Q_{4}=5.9316 & Q_{5}=6.1071 & Q_{6}=7.4454 \\
Q_{7}=1.8377 & Q_{8}=2.2122 & Q_{9}=0.0746 \\
Q_{10}=1.3863 & &
\end{array}
$$

Let the impact factor of the journal be, $F=2$. Let the journal have 25 reviewers with g-index given respectively as $1,2,5,10,4,6,9$, $10,5,1,1,4,5,6,7,8,9,5,20,15,6,5,10,9,11$.

Then

$$
\begin{gathered}
\frac{1}{k} \sum_{i=1}^{k} R_{i}=\frac{1}{25}(1+2+5+10+4+6+9+10+5+1+1+4+5+6+7+8+9 \\
+5+20+15+6+5+10+9+11)=1.1915 \\
Q_{T}=\sum_{j=1}^{m} Q_{j}=3.2343+3.9613+4.5135+5.9316+6.1071+7.4454+1.8377 \\
+2.2122+0.0746+1.3863=36.7040
\end{gathered}
$$

Quality of a single issue of a journal is given by

$$
\begin{aligned}
J= & {\left[\log (1+(1+F) Q] \times \frac{1}{k} \sum_{i=1}^{k} R_{i}=[\log (1+(1+2) \times 36.7040)] \times 1.1915\right.} \\
= & 5.6126
\end{aligned}
$$

\section{CONCLUSION}

Due to the high necessity of quality index for individual journal articles, authors and journals, this paper has introduced new measures of quality for journals, authors and individual journal articles.

\section{ACKNOWLEDGMENTS}

The authors sincerely thank the NeST for giving permission to do the research and all other who directly or indirectly helped in doing this research. Dr. R. Rajesh, Assistant Professor (on leave), Bharathiar University, is currently working as Senior Research Associate at NeST and he is thankful to Bharathiar University for providing long leave to work at NeST.

\section{REFERENCES}

[1] M. Amin and M. Mabe, "Impact factors: Use and abuse," Perspectives in Publishing 1 (2000), 16.

[2] Judit Bar-Ilan, "Which h-index? A comparison of WoS, Scopus and Google Scholar," Scientometrics, Vol. 74, No. 2 (2008), 257-271 
[3] Carl T. Bergstrom, Jevin D. West, and Marc A. Wiseman,"The Eigenfactor Metrics," The Journal of Neuroscience, November 5, 2008 28(45):11433-11434

[4] Clint D. Kelly and Michael D. Jennions, "The $h$ index and career assessment by numbers," Trends in Ecology and Evolution, Vol.21, No.4, April 2006

[5] Lutz Bornmann, Hans-Dieter Daniel,"What Do We Know About the h Index?," Journal of the American Society for Information Science and Technology, 58(9):13811385, 2007

[6] Cole S, Cole JR, Simon GA., "Chance and consensus in peer review," Science, 1981;214:881-6.

[7] Ernst E, Saradeth T, Resch KL, Drawbacks of peer review, Nature, 1993;363:296. [Medline]

[8] Garfield E., "Citation analysis as a tool in journal evaluation," Science, 1972;178:471-9.

[9] Gordon MD., "How authors select journals: a test of the reward maximization model of submission behavior," Social Studies of Science, 1984;14:27-43.

[10] Hansen HF, Jrgensen BH., "Styring af forskning: kan forskningsindikatorer anvendes?," Frederiksberg, Denmark: Samfundslitteratur, 1995.

[11] J.E. Hirsch, "An index to quantify an individuals scientific research output," PNAS, 102(46): 16569-72, 15 November 2005.

[12] "Does the h-index have predictive power?," PNAS, 104(49): 19193-8, 26 November 2007.

[13] Maffulli N., "More on citation analysis," Nature, 1995; 378:760.

[14] Magri M-H, Solari A., "The SCI Journal Citation Reports: a potential tool for studying journals? I. Description of the JCR journal population based on the number of citations received, number of source items, impact factor, immediacy index and cited half-life," Scientometrics, 1996;35:93-117.

[15] Moed HF, Van Leeuwen TN., "Impact factors can mislead," Nature, 1996; 381:186.

[16] Moed HF, Van Leeuwen TN, Reedijk J., "A critical analysis of the journal impact factors of Angewandte Chemie and the Journal of the American Chemical Society. Inaccuracies in published impact factors based on overall citations only," Scientometrics, 1996;37:105-16.

[17] Erhard Rahm, "Comparing the scientific impact of conference and journal publications in computer science," Information Services \& Use, 28 (2008) 127128.

[18] Mike Rossner, Heather Van Epps, and Emma Hill, "Show me the data," JCB, pp. 1092-1091, December 17, 2007

[19] Per O Seglen, "Why the impact factor of journals should not be used for evaluating research," BMJ, 1997;314:497 (15 February).

[20] Seglen PO., "Evaluation of scientists by journal impact",. In: Weingart $\mathrm{P}$, Sehringer R, Winterhager $\mathrm{M}$, eds. representations of science and technology. Leiden: DSWO Press, 1992;240-52.

[21] Seglen PO., "Causal relationship between article citedness and journal impact," J. Am. Soc. Information Sci., 1994;45:1-11.

[22] Sengupta IN., "Three new parameters in bibliometric research and their application to rerank periodicals in the field of biochemistry," Scientometrics, 1986;10:235-42.

[23] Vinkler P., "Evaluation of some methods for the relative assessment of scientific publications," Scientometrics, 1986;10:157-77. 